Provided for non-commercial research and education use. Not for reproduction, distribution or commercial use.

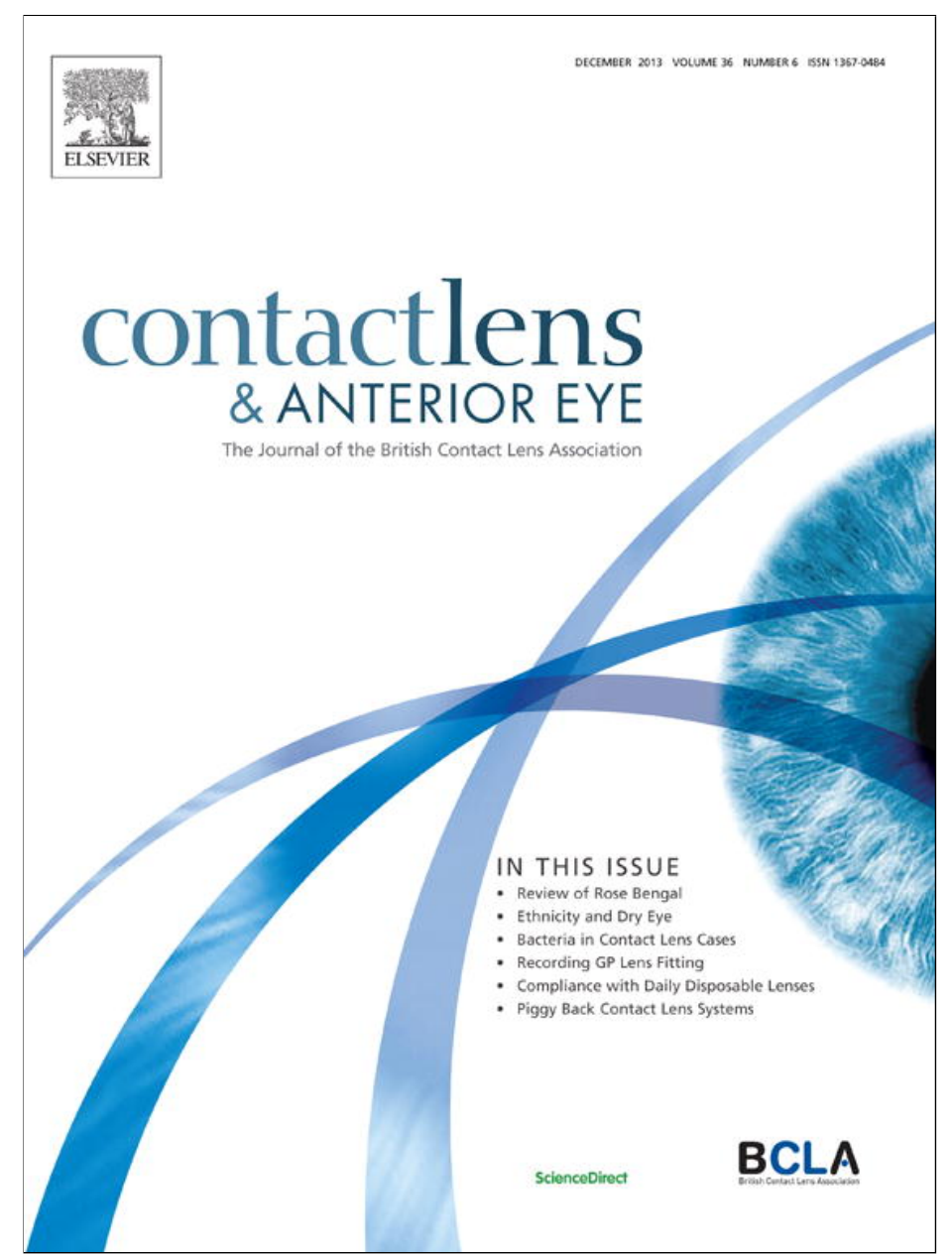

This article appeared in a journal published by Elsevier. The attached copy is furnished to the author for internal non-commercial research and education use, including for instruction at the authors institution and sharing with colleagues.

Other uses, including reproduction and distribution, or selling or licensing copies, or posting to personal, institutional or third party websites are prohibited.

In most cases authors are permitted to post their version of the article (e.g. in Word or Tex form) to their personal website or institutional repository. Authors requiring further information regarding Elsevier's archiving and manuscript policies are encouraged to visit:

http://www.elsevier.com/authorsrights 


\title{
Multi-site clinical assessment of Complete Revitalens MPDS in 2981 contact lens wearers across Europe and USA
}

\author{
J.M. González-Méijome*, A.C. da Silva, M. Faria-Ribeiro, D. Lopes-Ferreira, \\ S.C. Peixoto-de-Matos \\ Clinical \& Experimental Optometry Research Lab, Center of Physics, University of Minho, Braga, Portugal
}

\section{A R T I C L E I N F O}

\section{Article history:}

Received 22 December 2012

Received in revised form 30 March 2013

Accepted 31 May 2013

\section{Keywords:}

MPDS

Contact lens care

Silicone-hydrogel

Comfort

Dryness

\begin{abstract}
A B S T R A C T
Purpose: To evaluate ocular response and subject acceptance of a new multipurpose disinfecting solution (MPDS), Complete Revitalens (RevitaLens OcuTec in the US Market), for soft contact lens care in a large "real practice" setting.

Method: This is an international multi-center, open-label assessment carried out in 10 countries across Europe and in the USA. Up to 10 subjects who were currently wearing soft contact lenses for at least 1 year and using a MPS as a lens care system were included at each investigational site.

Results: Data were collected from 996 European and 1985 American wearers, 75\% of those patients wore silicone hydrogel contact lenses. Approximately 94\% found the new MPDS "somewhat more effective" to "much more effective" in keeping contact lenses feeling clean and $88 \%$ found the new MPDS to be somewhat more effective to much more effective in keeping their lenses feeling comfortable in the evening. Over 93\% reported an improvement in vision clearness in the evening after approximately 1 month while using the new MPDS. Wearers with grade 2,3 or 4 of severity decreased by $11.3,6.4$ and $9.8 \%$ over 1 month period for redness, burning and irritation, respectively. After approximately 1 month $83 \%$ of wearers declared that they would prefer to use the new MPDS.

Conclusions: Over $88 \%$ felt their lenses were somewhat to much more comfortable at the end-of-day and 94\% found the new MPDS to be somewhat to much more effective in keeping their lenses feeling clean compared to their previous care system.
\end{abstract}

(c) 2013 British Contact Lens Association. Published by Elsevier Ltd. All rights reserved.

\section{Introduction}

Multipurpose disinfecting solutions (MPDS) for contact lens care have been one of the areas in the contact lens industry that has experienced more innovations. Part of these advances have been driven by several contamination outbreaks, but also by the challenges imposed by the new generations of silicone hydrogel ( $\mathrm{Si}-\mathrm{Hy}$ ) contact lenses and the compatibility issues linked to the interaction between MPDS constituents, the contact lens materials and the ocular surface [1,2].

The commitment of developing more efficacious MPDS that are also fully compatible with the ocular surface when used in combination with the growing category of Si-Hy contact lens materials puts a lot of pressure on the industry to develop high performance products. Recently, new MPDS that attempt to improve the safety and efficacy of soft CL have been developed and their results are promising either from the antimicrobial and/or clinical performance perspective [3-5].

In a recent study we have evaluated ex vivo dehydration, pre-lens tear stability, optical quality of the eye and subjective performance with two new double biocide MPDS including Complete Revitalens MPDS. The outcomes showed that both solutions provided excellent preservation of pre-lens tear film stability and consistent optical quality of the eye over 1 month of wear of Lotrafilcon B Si-Hy contact lens. Furthermore, voluntaries reported less than 1.5 points of reduction in end-of-day dryness which is remarkable considering that all of them were neophytes.

The present study was designed to evaluate in the "real practice environment" the ocular safety, efficacy and subject acceptance of Complete Revitalens MPDS, also known as RevitaLens OcuTec in the USA, in subjects currently wearing soft contact lenses and using a multipurpose contact lenses solution.

\section{Methods}

\footnotetext{
* Corresponding author at: CEORLab, Center of Physics (Optometry), School of Science, University of Minho, 4710-057 Gualtar, Braga, Portugal. Tel.: +351 253604072. E-mail address: jgmeijome@fisica.uminho.pt (J.M. González-Méijome).
}

This was an international multi-centre, open-label assessment conducted across Europe and USA. According to the protocol, up to 
Table 1

Composition of MPDS used in the study.

\begin{tabular}{ll}
\hline & COMPLETE $^{\circledR}$ Revitalens MPDS \\
\hline Preservative & Polyquaternium-1: 0.0003\% Alexadine \\
& $0.00016 \%$ \\
Buffer & Boric acid, sodium borate decahydrate, \\
& trisodium citrate dehydrate. \\
Chelating agent & EDTA \\
Surfactant & Tetronic 904 \\
Tear film electrolytes & Sodium chloride \\
Other & Rub-and-rinse \\
\hline
\end{tabular}

10 subjects currently wearing soft contact lenses and using a multipurpose contact lens solution, were included in this assessment by each participating Eye Care Professional and asked to change from their habitual care system to Complete Revitalens MPDS for the duration of the study. Technical details of the MPDS are available in Table 1.

Inclusion criteria required that prior to enrollment subjects had a history of wearing soft contact lenses for 12 months or more; lens refit subjects had worn the same soft contact lens brand, fit and refractive correction for 6 months or more; subjects had been using a multipurpose contact lens solution to care for their lenses. Exclusion criteria included subjects with a history of wearing soft contact lenses for less than 12 months; refit subjects who had worn their current soft contact lens brand, fit and correction for less than 6 months; and subjects who were using a hydrogen peroxide cleaning system.

Each Eye Care Professional examined the subjects according to his/her standard clinical practice. At the initial and final visits (approximately 30 days from initial visit) the Eye Care Professional recorded the overall severity of the subject ocular sign (redness) and symptoms (burning, irritation, and discomfort). In addition, at each visit the Eye Care Professional and/or their staff asked each subject a series of questions regarding the multipurpose contact lens solution they were using.

After explaining the nature of the assessment the wearers were asked to sign the consent form. Two questionnaires were administered to volunteers; one at the baseline visit when the new MPDS solution was dispensed (baseline visit) and another one after approximately 1 month of use of the new MPDS (follow-up visit). At baseline and at follow-up visit, an examination was performed to evaluate the status of the ocular surface with special attention to conjunctival redness graded from 0 to 4 according to Efron Grading Scales.

Statistical analysis was conducted using JMP software v.6.0 (JMP, Cary, NC). Demographic data and study variables were analyzed on subjects who complete the initial and follow-up visit. Descriptive statistics (i.e., mean, standard deviation, etc.) were obtained for all continuous variables and frequencies for all categorical variables collected in this trial. Efficacy was assessed by evaluating changes from baseline for all variables. Subject acceptance was assessed by evaluating subjects' evaluation of Complete Revitalens MPDS. Statistical significance was set for values of $\alpha<0.05$.

\section{Results}

A total of 2981 wearers were assessed in this study. Distribution of wearers across countries, demographic information and contact lens experience for the sample collected are listed in Table 2. About $70 \%$ of the surveyed volunteers were female and approximately $69 \%$ of all wearers had worn lenses for over 5 years before enrollment in this assessment.

Of the 2666 wearers whose previous contact lenses were identified, $74.8 \%$ were wearing silicone-hydrogel contact lenses and $25.2 \%$ were wearing conventional hydrogel contact lenses; for 315 wearers $(10.6 \%)$ the habitual contact lenses could not be identified. Reported wearing time at baseline with their habitual contact lenses was $12-15 \mathrm{~h} /$ day in $51.3 \%$ of the wearers, while $17.2 \%$ reported that they wore their lenses for over $15 \mathrm{~h} /$ day. Regarding their habitual care system, 33.9\% used Optifree Replenish, 16.3\% Optifree Express, 9.1\% ReNu Fresh, 5.4\% Biotrue, 5.2\% ReNu Multiplus, $4.6 \%$ Solocare and $2.9 \%$ ReNu Sensitive accounting altogether for $77.4 \%$; further $16.1 \%$ were identified as generic/store brand while the remaining $6.5 \%$ were other products accounting for less than $2 \%$ each. About $35 \%$ of the wearers enrolled reported to use some kind of rewetting drops at their baseline visit.

Volunteers used the new MPDS for an average of $37.8 \pm 18$ days at the moment of the final assessment and the results were compared to the initial responses given at the baseline visit.

Fig. 1 shows the change in conjunctival redness as assessed by the practitioner as well as burning sensation and irritation selfreported by the wearer. There was a significant increase in the proportion of wearers with grade 0 of ocular redness, motivated by also significant reduction in wearers with grade 1 and grade 2 levels of ocular redness, burning and irritation experienced with the new solution. There was a statistically significant reduction in redness, burning and irritation from baseline to the follow-up visit after approximately 1 month ( $p<0.001$, paired $T$-test).

Fig. 2 shows the comparative perception of the wearer regarding cleaning efficacy and comfort with the new solution against their previous solution. In this case, 93.5\% reported that they felt the new solution was somewhat effective to much more effective in keeping their lenses feeling clean while $90.2 \%$ of the volunteers enrolled reported that they felt the new solution was somewhat effective to much more effective in keeping their lenses feeling comfortable compared to the solution they were previously using. There was a statistically significant reduction in ocular discomfort from baseline to the follow-up visit after approximately 1 month $(p<0.001$, paired

Table 2

Demographic data and contact lens wearing experience by country.

\begin{tabular}{|c|c|c|c|c|c|}
\hline Country & $n$ & Age & Female & $\begin{array}{l}\text { Wearing } \mathrm{CL} \leq 5 \\
\text { years }\end{array}$ & $\begin{array}{l}\text { Wearing } \mathrm{CL}>5 \\
\text { years }\end{array}$ \\
\hline Austria & 42 & $34.7 \pm 13$ & $61.9 \%$ & $57.2 \%$ & $42.9 \%$ \\
\hline Belgium & 14 & $42.6 \pm 19$ & $80.0 \%$ & $13.4 \%$ & $86.7 \%$ \\
\hline England & 39 & $45.0 \pm 14$ & $66.7 \%$ & $33.3 \%$ & $66.7 \%$ \\
\hline France & 175 & $38.5 \pm 14$ & $65.9 \%$ & $40.0 \%$ & $60.0 \%$ \\
\hline Germany & 287 & $34.8 \pm 12$ & $68.3 \%$ & $48.8 \%$ & $51.2 \%$ \\
\hline Italy & 248 & $34.6 \pm 12$ & $64.1 \%$ & $47.2 \%$ & $52.8 \%$ \\
\hline Portugal & 8 & $21.8 \pm 2$ & $75.0 \%$ & $87.5 \%$ & $12.5 \%$ \\
\hline Spain & 159 & $32.6 \pm 13$ & $69.8 \%$ & $46.6 \%$ & $53.4 \%$ \\
\hline Sweden & 10 & $25.3 \pm 6$ & $50.0 \%$ & $40.0 \%$ & $60.0 \%$ \\
\hline Switzerland & 12 & $31.3 \pm 10$ & $58.3 \%$ & $33.3 \%$ & $66.7 \%$ \\
\hline USA & 1985 & $36.2 \pm 13$ & $71.8 \%$ & $24.3 \%$ & $75.7 \%$ \\
\hline Average total & 2979 & $35.9 \pm 13$ & $71.1 \%$ & $31.4 \%$ & $68.6 \%$ \\
\hline
\end{tabular}




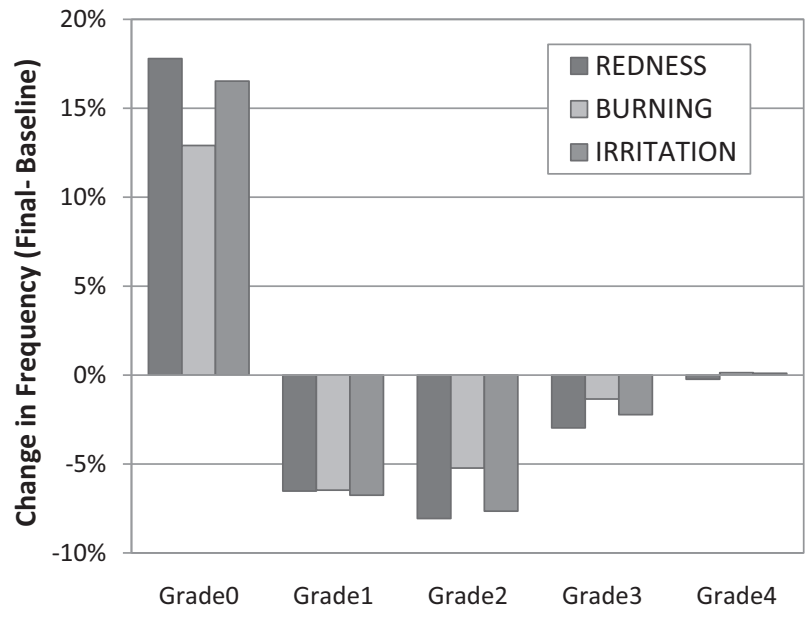

Fig. 1. Change in grades of redness, burning and irritation after approximately 1 month with Complete Revitalens MPDS.

$T$-test). This is noteworthy considering that the levels of comfort reported by the wearers at enrollment was considered comfortable to very comfortable by $85.9 \%, 73.5 \%$ and $41.4 \%$ of the volunteers in the morning, afternoon and evening, respectively (Fig. 3A). After approximately 1 month using the new solution $93.4 \%, 91.9 \%$ and $87.9 \%$ of the volunteers reporting they felt their lenses somewhat more comfortable to much more comfortable with the new solutions in the morning, afternoon and evening, respectively (Fig. 3B).

The wearers also reported their subjective sensation concerning clearness of vision with their lenses at baseline and after approximately 1 month using the new MPDS. Again, despite the good subjective perception of vision clearness at study entry (Fig. 4A), 96.8\%, 95.3\% and 93\% showed an improvement in this parameter in the morning, afternoon and evening, respectively after approximately 1 month using the new MPDS (Fig. 4B).

\section{Discussion}

The outcomes from this study represent a singular opportunity for eye care professionals to be able to participate in the assessment of ocular safety, efficacy and subject acceptance of a new MPDS product and provide a "real-world" insight regarding the acceptance of these products in a large cohort.

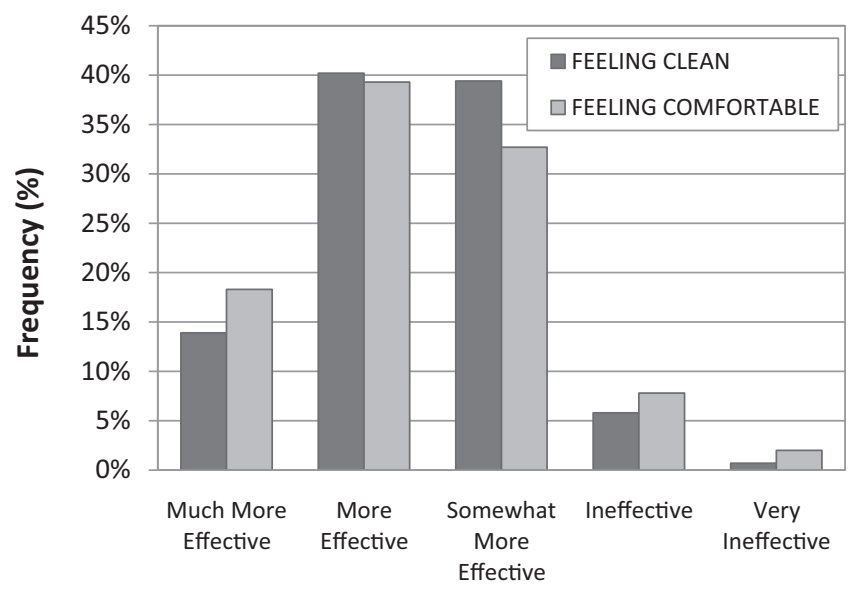

Fig. 2. Change in feeling clean and comfortable after approximately 1 month with Complete Revitalens MPDS.
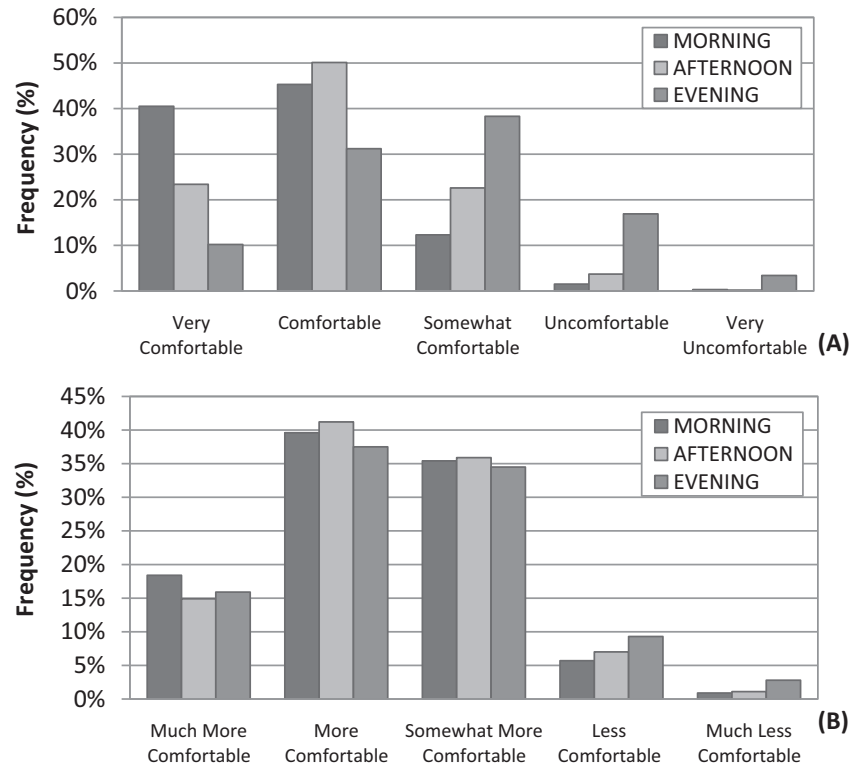

Fig. 3. Comfort at baseline with the wearer's MPS (A) and change in comfort after approximately 1 month with Complete Revitalens MPDS (B).

Multipurpose disinfection solutions have dominated the global market for the last decade, accounting for over $70 \%$ of all contact lens wearers in most countries. Despite the increase in the global trends for daily disposable soft contact lenses [6] frequent replacement continues to represent the majority of fittings [7], thus requiring a care system. MPDS have evolved to a convenient form of cleaning, disinfecting and conditioning contact lenses in a single product. Indeed, the data from the Contact Lens Fitting Survey Consortium show that over $80 \%$ of the care systems prescribed consist of multipurpose solutions [7].

An effective and safe care solution is fundamental for a satisfactory contact lens wear experience, but their components can also interact with the contact lens material. Si-Hy contact lenses are thought to interact in a different way with MPDS such that can be a cause of ocular discomfort, irritation and even inflammation
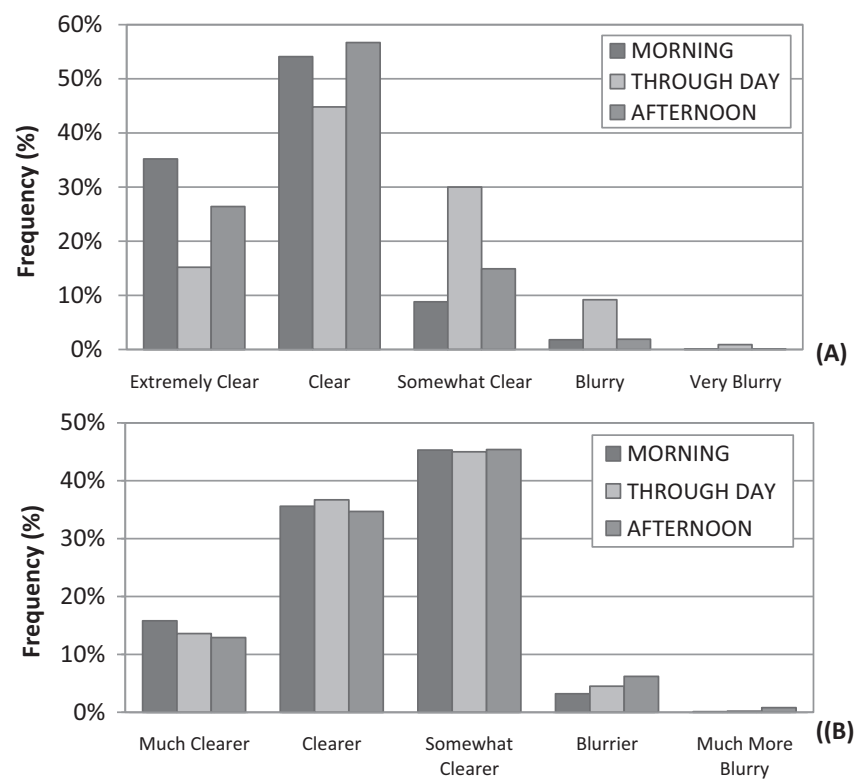

Fig. 4. Subjective vision at baseline with wearer's previous MPS (A) and change in vision self-perception after approximately 1 month with Complete Revitalens (B). 
[8]. Thus, it is of primary importance to assess the compatibility of new products with these types of materials. As a result of their unique composition and the need of surface treatments in some cases, Si-Hy contact lenses have a pattern of deposit adsorption much different from conventional hydrogels $[9,10]$. The efficacy of MPS care systems in cleaning the contact lenses is in agreement with a study conducted by Nichols [11] showing that less than $10 \%$ of wearers exhibited clinically significant deposition with a silicone hydrogel contact lens combined with Complete MoisturePlus. The present study is particularly interesting because of the population sampled consisted mostly of Si-Hy contact lens wearers (66.7\% of the total sample or $74.8 \%$ of all identifiable lenses). Moreover, the sample size capture in this study should represent well the actual clinical population and the outcomes that might be expected by clinicians when choosing to change the MPDS to wearers wearing silicone hydrogel contact lens materials. Lebow and Schachet evaluated 3 different multipurpose solutions combined with two different conventional hydrogel high water content contact lens materials [12]. Wearers reported a $57.1 \%$ preference for one of the care regimes evaluated. It is not possible to compare our preference results with those of the study mentioned considering the different nature of each one. In a different study, Garofalo et al. evaluated the symptoms reported by contact lens wearers with 4 different care systems [13]. Three of the solutions used in that study (Opti-Free Express, ReNu Multiplus and Solo-Care) account for $45 \%$ of all the care systems reported in the present study.

The main outcomes reported here highlight the good performance of the new MPDS for two of the most critical aspects of contact lens wear; wearer's comfort and vision self-perception. A large proportion of the cohort reported an improvement in comfort with the new care system. Our previous study showed a slight drop of 1.5 points in a 10 point Visual Analogue Scale in dryness after $10 \mathrm{~h}$ of wear from the dispensing visit to the 30 days visit in neophyte wearers prescribed with a Si-Hy lens combined with Complete Revitalens MPDS in one eye and Biotrue in the contralateral eye; there were no significant differences between solutions despite their different composition and the incorporation of hyaluronic acid as wetting agent in the former one. Campbell et al. compared the comfort at insertion and removal with a new MPDS incorporating a wetting agent. The authors found improvement in comfort and decreasing deposits [14]. Guillon and Maisa had also reported benefits from incorporating HPMC in the solution in terms of improving comfort [15]. However, in our previous stud we did not observe a significant difference in comfort, wettability or optical quality with Biotrue containing hyaluronic acid compared to Revitalens [16]. The results of the present study reinforce the fact that this new MPDS is able to enhance comfort in successful contact lens wearers despite not incorporating any specific comfort agent in the formulation.

Regarding vision perception, there was also a remarkable improvement in the subjective perception of vision clearness. This is not surprising considering our previous results where the combination of the new MPDS products combined with a Si-Hy material provided a stable pre-lens tear film resulting in a consistent uniform pattern of optical aberrations over the month of study.

It is important to notice that all contact lens wearers included in the study were successfully using their lenses. Despite this, the new MPDS was able to improve comfort in most of the wearers to a certain extent and quite significantly in almost $50 \%$ of the sample. This is, is in agreement with Corbin et al. who showed that switching to a new MPDS system might improve significantly lens comfort even in successful contact lens wearers. It is important to highlight that Corbin's study design was very similar to the present study, although with a much smaller sample of 109 wearers [17]. Both studies support the evidence that it is safe to switch to a new MPDS with minimal risks of non-satisfactory outcomes.
A limitation of this study is the fact that the placebo effect cannot be ruled out as the wearers and clinicians were not masked and no control group was used. This can influence the subject's and even the practitioner perception about the new product, thus rendering an improvement with the new product, part of which might not be directly related with an objective benefit provided by the product itself. Despite this, these results can be considered as representative of the clinical situation with the exception made to the fact that in this case the wearer was not charged for the new solution, which might again affect the positive opinion about the product; in this context is significant however, that over $80 \%$ of the wearers would be willing to purchase the new product over the previous one they were using before. Finally, there might be differences in the criteria to judge ocular redness but this should not affect the comparison between dispensing and follow-up visit by the same practitioner.

In summary, the present report offers us a large amount of information regarding the contact lens care habits followed by soft contact lens wearers regarding their care systems and contact lens wearing experience. According to these results, the new MPDS under evaluation was able to show a significant improvement in comfort in wearers using some of the care systems more commonly used world-wide. Furthermore, the solution seems to produce satisfactory clinical results when combined with the most frequently prescribed silicone hydrogel contact lenses which represented over $2 / 3$ of the prescriptions considered in this study. However, these results might not be directly applied to the real clinical situation considering some methodological limitations that might overestimate the positive attitude of the subject towards a new product provided free of charge. Thus, the placebo effect could not be discarded with this protocol design and future randomized and masked studies might evaluate the potential benefit pointed in this report.

\section{Acknowledgements}

The authors wish to thank the eye care practitioners participating in this study across Europe and USA. The D'Ellis Group for the statistical support and Rafael Guerrero and Will Heydorn from Abbott Medical Optics, Inc. for their contributions in planning and coordinating the European and American branches of the study, respectively. The authors declare no proprietary or financial interest in any of the materials mentioned in this article. This study has been sponsored by Abbott Medical Optics Inc. These results were presented to the American Academy of Optometry Boston, 12-15th, 2011.

\section{References}

[1] Santodomingo-Rubido J, Barrado-Navascués E, Rubido-Crespo MJ, Sugimoto K Sawano T. Compatibility of two new silicone hydrogel contact lenses with three soft contact lens multipurpose solutions. Ophthalmic and Physiological Optics 2008;28(4):373-81.

[2] Chalmers RL, Keay L, McNally J, Kern J. Multicenter case-control study of the role of lens materials and care products on the development of corneal infiltrates. Optometry and Vision Science 2012;89(3):316-25.

[3] Zhu H, Ding A, Bandara M, Willcox MD, Stapleton F. Broad spectrum of antibacterial activity of a new multipurpose disinfecting solution. Eye \& Contact Lens 2007;33(6 Pt 1):278-83.

[4] Reindel W, Cairns G, Merchea M. Assessment of wearer and practitioner satisfaction with Biotrue ${ }^{\mathrm{TM}}$ multi-purpose solution for contact lenses. Contact Lens \& Anterior Eye 2010;33(Suppl. 1):S12-7.

[5] Kilvington S, Huang L, Kao E, Powell CH. Development of a new contact lens multipurpose solution: comparative analysis of microbiological, biological and clinical performance. Journal of Optometry 2010;3: 134-42.

[6] Efron N, Morgan PB. Woods CA; The International Contact Lens Prescribing Survey Consortium. An international survey of daily disposable contact lens prescribing. Clinical and Experimental Optometry 2013;96: 58-64. 
[7] Morgan P, Woods CA, Tranoudis I, Helland M, Efron N, Grupcheva CN, et al. International contact lens prescribing in 2011. Contact Lens Spectrum 2012;1:26-31.

[8] Carnt N, Jalbert I, Stretton S, Naduvilath T, Papas E. Solution toxicity in soft contact lens daily wear is associated with corneal inflammation. Optometry and Vision Science 2007;84(4):309-15.

[9] Subbaraman LN, Glasier MA, Senchyna M, Sheardown H, Jones L. Kinetics of in vitro lysozyme deposition on silicone hydrogel, PMMA, and FDA groups I, II, and IV contact lens materials. Current Eye Research 2006;31(10):787-96.

[10] Carney FP, Nash WL, Sentell KB. The adsorption of major tear film lipids in vitro to various silicone hydrogels over time. Investigative Ophthalmology and Visual Science 2008;49(1):120-4.

[11] Nichols JJ. Deposition rates and lens care influence on galyfilcon A silicone hydrogel lenses. Optometry and Vision Science 2006;83(10):751-7.

[12] Lebow KA, Schachet JL. Evaluation of corneal staining and patient preference with use of three multi-purpose solutions and two brands of soft contact lenses. Eye \& Contact Lens 2003;29(4):213-20.
[13] Garofalo RJ, Dassanayake N, Carey C, Stein J, Stone R, David R. Corneal staining and subjective symptoms with multipurpose solutions as a function of time. Eye \& Contact Lens 2005;31(4):166-74.

[14] Campbell R, Kame G, Leach N, Paul M, White E, Zigler L. Clinical benefits of a new multipurpose disinfecting solution in silicone hydrogel and soft contact lens users. Eye \& Contact Lens 2012;38(2):93-101.

[15] Guillon M, Maissa C. Clinical acceptance of two multipurpose solutions: MPS containing HPMC versus citrate-based MPS without rubbing. CLAO Journal 2002;28(4):186-91.

[16] González-Méijome JM, da Silva AC, Neves H, Lopes-Ferreira D, Queirós A, Jorge J. Clinical performance and ex vivo dehydration of silicone hydrogel contact lenses with two new multipurpose solutions. Contact Lens \& Anterior Eye 2013;36:86-92.

[17] Corbin GS, Bennett L, Espejo L, Carducci S, Sacco A, Hannigan R, et al. A multicenter investigation of OPTI-FREE RepleniSH multi-purpose disinfecting solution impact on soft contact lens wearer comfort. Clinical Ophthalmology 2010;4:47-57. 